\title{
Laser development for LISA
}

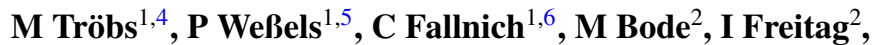 \\ S Skorupka $^{3}$, G Heinzel ${ }^{3}$ and K Danzmann ${ }^{3}$ \\ ${ }^{1}$ Laser Zentrum Hannover, Hollerithallee 8, D-30419 Hannover, Germany \\ 2 Innolight GmbH, Garbsener Landstr. 10, D-30419 Hannover, Germany \\ ${ }^{3}$ Max Planck Institute for Gravitational Physics, Callinstr. 38, D-30167 Hannover, Germany \\ E-mail: michael.troebs@aei.mpg.de
}

Received 14 September 2005, in final form 19 December 2005

Published 28 March 2006

Online at stacks.iop.org/CQG/23/S151

\begin{abstract}
The two most promising configurations for the LISA laser are a stand-alone diode-pumped nonplanar ring oscillator (NPRO) or a fibre amplifier seeded by a low-power NPRO. The stand-alone laser was stabilized in frequency to a ULE cavity and in power to an electronic reference. For the first time the LISA requirement of relative power noise below $2 \times 10^{-4} / \mathrm{Hz}^{1 / 2}$ was fulfilled in the whole frequency range from $0.1 \mathrm{mHz}$ to $1 \mathrm{~Hz}$. The LISA goal of frequency noise below $30 \mathrm{~Hz} / \mathrm{Hz}^{1 / 2}$ was achieved for frequencies above $3 \mathrm{mHz}$. As a first step in the characterization of an oscillator-amplifier system, the excess frequency noise of an ytterbium-doped fibre amplifier was measured. For frequencies between $0.1 \mathrm{mHz}$ and $1 \mathrm{~Hz}$ the excess noise was measured to be below $0.1 \mathrm{~Hz} / \mathrm{Hz}^{1 / 2}$, which is significantly below the free-running frequency noise of NPROs.
\end{abstract}

PACS number: 42.55.Xi

(Some figures in this article are in colour only in the electronic version)

\section{Introduction}

The Laser Interferometer Space Antenna (LISA) consists of three satellites forming an equilateral triangle in heliocentric orbits. LISA requires single-frequency laser sources with at least $1 \mathrm{~W}$ of output power [1]. Due to celestial mechanics, armlength differences of the order $1 \%(50000 \mathrm{~km})$ will be expected and an effective laser frequency stability in the $\mu \mathrm{Hz} / \mathrm{Hz}^{1 / 2}$ range is required. Multiple measures are necessary to achieve this: frequency stabilization to optical cavities [2], offset phase locking, arm-locking [3] and time-delay interferometry [4].

\footnotetext{
4 Present address: Max Planck Institute for Gravitational Physics, Callinstr. 38, D-30167 Hannover, Germany.

5 Present address: Innolight GmbH, Garbsener Landstr. 10, D-30419 Hannover, Germany.

6 Present address: Physikalisch-Technische Bundesanstalt, Bundesallee 100, D-38116 Braunschweig, Germany.
} 


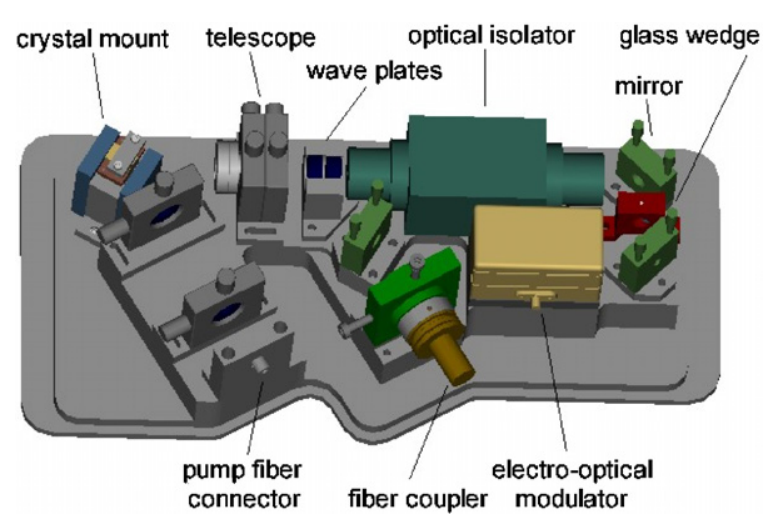

Figure 1. Schematic setup of the single-stage laser demonstrator.

For the phase measurement a portion of light is reflected off the test masses. The reflected light produces forces on the test masses and fluctuating light powers produce fluctuating forces. For a $2 \mathrm{~kg}$ test mass and $100 \mu \mathrm{W}$ of light a relative power stability of better than $2 \times 10^{-4} / \mathrm{Hz}^{1 / 2}$ is required.

There are two promising laser candidates for LISA: a single-stage nonplanar ring oscillator (NPRO) [5] or a low-power NPRO together with a fibre amplifier. We report on a laser demonstrator for LISA based on a single-stage NPRO, power stabilization and frequency stabilization results as well as noise investigations in a two-stage laser system using a Ybdoped fibre amplifier.

\section{Single-stage laser}

The laser demonstrator for LISA was based on an NPRO that was longitudinally diode pumped through the crystal front facet. In order to obtain high output powers, the thermally induced lens of the NPRO was partially compensated by a concave front facet with $-1500 \mathrm{~mm}$ radius of curvature. The region directly behind the front facet was made of undoped YAG to avoid spatial hole burning and to ensure single-frequency operation [6].

Figure 1 shows the schematic setup of the LISA laser demonstrator. The pump light from fibre coupled laser diodes (for details see below) was delivered by a multimode fibre (100 $\mu \mathrm{m}$ core diameter, $N A=0.22)$, collimated with a lens $(f=30 \mathrm{~mm})$ and focussed into the laser crystal with a second lens $(f=25 \mathrm{~mm})$. The laser radiation was transmitted through a telescope that generated a collimated beam with $1 \mathrm{~mm}$ beam diameter. A quarter-wave plate and a half-wave plate were used to transform the elliptically polarized light into linearly polarized light and to adjust the polarization axis to that of the subsequent optical isolator that protected the laser from backreflections. Two mirrors were used to align the light through the electro-optical modulator (EOM, model 4003 by New Focus) that was required for frequency stabilization. Optionally, a glass wedge could be placed between the two mirrors to couple out light without phase modulation for a beat measurement. A third mirror was used to couple the light into a single-mode polarization-maintaining fibre with angle-polished ends $\left(8^{\circ} \mathrm{FC}-\mathrm{APC}\right.$ type) to avoid backreflections and parasitic etalons. The fibre coupler (60SM-1-4-A4.5-03 by Schäfter \& Kirchhoff) was mounted on an XY-translation stage to centre the coupler to the optical beam. 


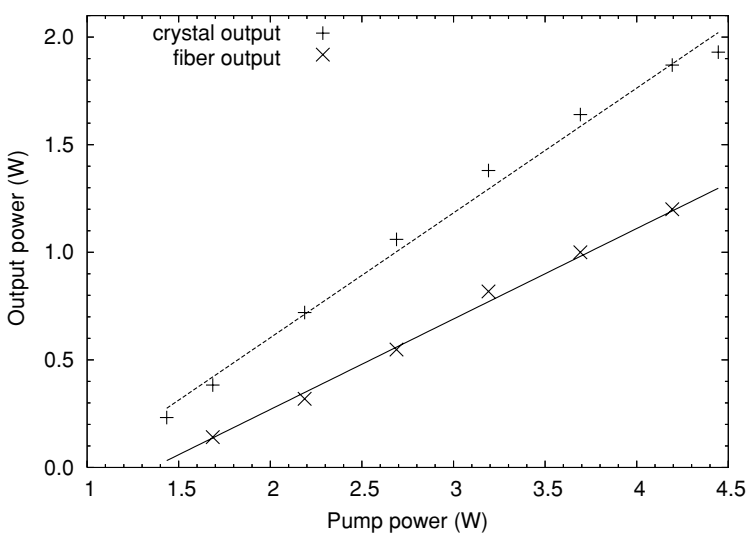

Figure 2. LISA laser demonstrator output power versus pump power.

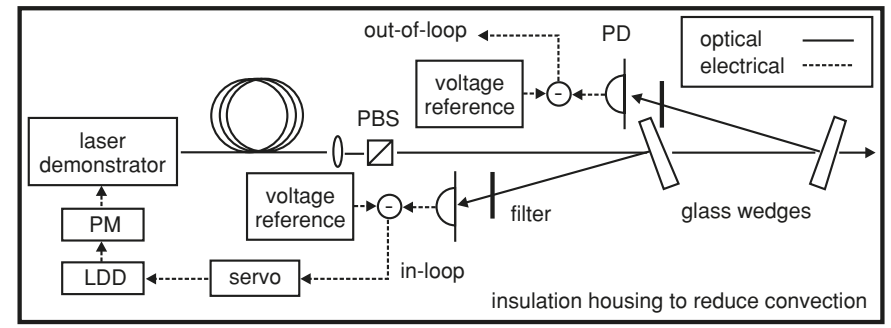

Figure 3. Power stabilization setup of the LISA laser demonstrator; Glan-air polarizer PBS, photodetector PD, laser diode driver LDD, pump module PM.

The pump module for the laser was manufactured by Brightpower [7]. It contained eight laser diodes (c-mounts) coupled into a multi-mode fibre $(100 \mu \mathrm{m}$ core diameter, $N A=0.22)$. The laser diodes shared a common water-cooled heat sink. At a cooling water temperature of $20{ }^{\circ} \mathrm{C}$ and $4 \mathrm{~W}$ of output power a wavelength of $806.5 \mathrm{~nm}$ with a full width at half maximum (FWHM) of $1.5 \mathrm{~nm}$ was measured. Two low-noise constant current laser diode drivers (Mephisto control electronics by Innolight modified to drive four diodes in series) were used to drive four laser diodes each.

Figure 2 shows the laser output power as a function of the pump power. Up to $1.9 \mathrm{~W}$ of output power has been obtained directly out of the laser crystal at $4.4 \mathrm{~W}$ of pump power. Out of the single-mode fibre, up to $1.2 \mathrm{~W}$ was measured.

\subsection{Power stabilization}

The NPRO-based laser demonstrator showed relative output power fluctuations up to $6 \times 10^{-2} / \mathrm{Hz}^{1 / 2}$ while LISA can only tolerate fluctuations below $2 \times 10^{-4} / \mathrm{Hz}^{1 / 2}$ [8, p 77]. Hence, an active power stabilization is required.

Figure 3 shows the power stabilization setup of the LISA laser demonstrator schematically. The light emitted from the laser demonstrator ( $1 \mathrm{~W}$ of output power) was collimated to a beam with $1 \mathrm{~mm}$ radius. A Glan-air polarizer (PBS) ensured purely s-polarized light. A fraction of the beam was sampled by reflection from an uncoated quartz glass wedge $\left(0.5^{\circ}\right.$ wedge 


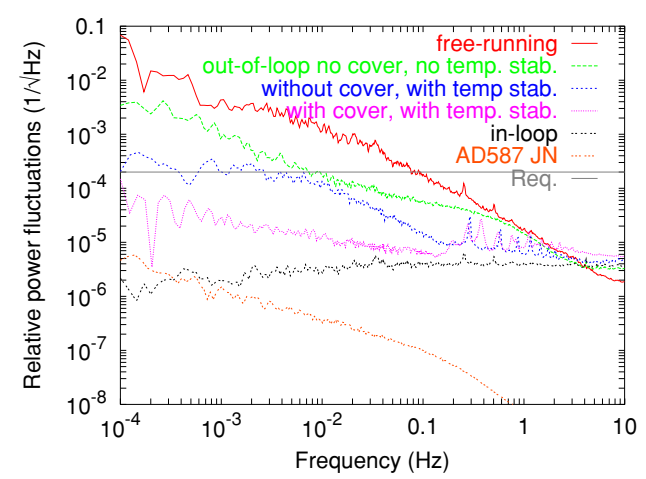

Figure 4. Relative power fluctuations under different operating conditions and internal noise of AD587JN voltage references.

angle) and directed to a temperature-stabilized photodetector. A pinhole (not shown) shielded the photodetector from the reflection of the back surface and a neutral density filter was used to reduce the photocurrent to values of about $0.5 \mathrm{~mA}$. The photodetector signal was subtracted from a filtered voltage reference (AD587) to generate the error signal (called inloop signal). A servo consisting of an integrator was used to feed back the error signal to the pump current of the laser demonstrator via the current modulation input of the laser diode driver. A second nominally identical error signal was generated independently and used as out-of-loop measurement.

Figure 4 shows the performance of the power stabilization for different operating conditions. The top trace shows the free-running power fluctuations of the LISA laser demonstrator. The trace below that shows the residual power fluctuations of the stabilized laser measured out-of-loop when the photodetectors were not temperature-stabilized. With temperature stabilization of the photodiodes, the curve labelled 'without cover, with temp. stab.' results as out-of-loop measurement. The spikes between $0.3 \mathrm{~Hz}$ and $3 \mathrm{~Hz}$ were caused by the pump module. The trace labelled 'with cover, with temp. stab.' was obtained by surrounding the experiment with a cover, thus reducing convection. A suppression of laser power fluctuations of up to three orders of magnitude has been measured. The lowest trace shows the internal noise of the voltage reference (AD587JN) that was utitilized and the trace labelled 'in-loop' shows the relative power fluctuations measured at the in-loop error signal.

With temperature stabilized photodetectors and shielding against convection, the LISA requirements of relative power noise below $2 \times 10^{-4} / \mathrm{Hz}^{1 / 2}$ could be fulfilled.

\subsection{Frequency stabilization}

Figure 5 shows a schematic of the optical setup. Beams from two laser systems were brought to interference on a fast photodiode and the photodiode signal was fed into a frequency counter (SRS 620 Option 01 by Stanford Research Systems). One laser system consisted of the LISA laser demonstrator and the other of a reference NPRO (labelled M2). The laser demonstrator was operated at an output power of $800 \mathrm{~mW}$, the reference laser M2 was operated at $100 \mathrm{~mW}$ of output power. Both lasers were locked to a resonance of its own thermally shielded reference cavity. From the LISA laser demonstrator, $400 \mu \mathrm{W}$ of optical power were transmitted through the reference cavity and $178 \mu \mathrm{W}$ through the second cavity from the reference NPRO.

The Pound-Drever-Hall reflection technique for frequency stabilization was used [9, 10]. A resonant EOM (see figure 1) generated $10 \mathrm{MHz}$ phase modulation sidebands around the 


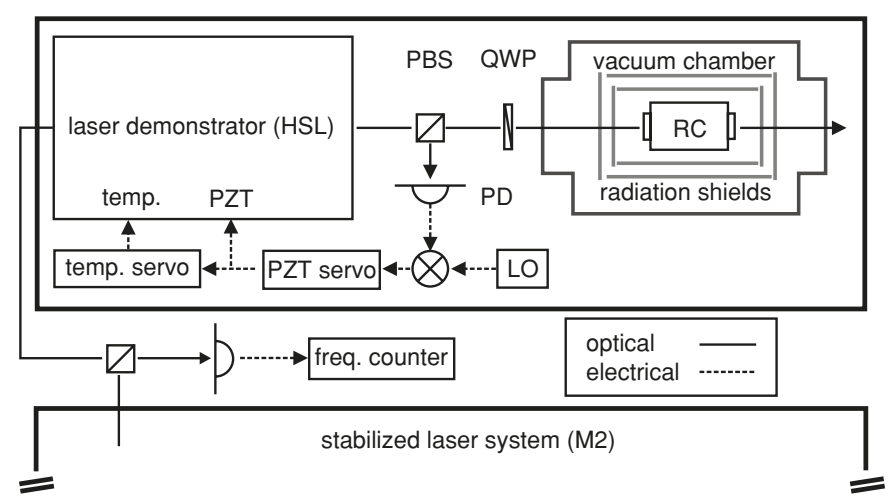

Figure 5. Setup for frequency stabilization.

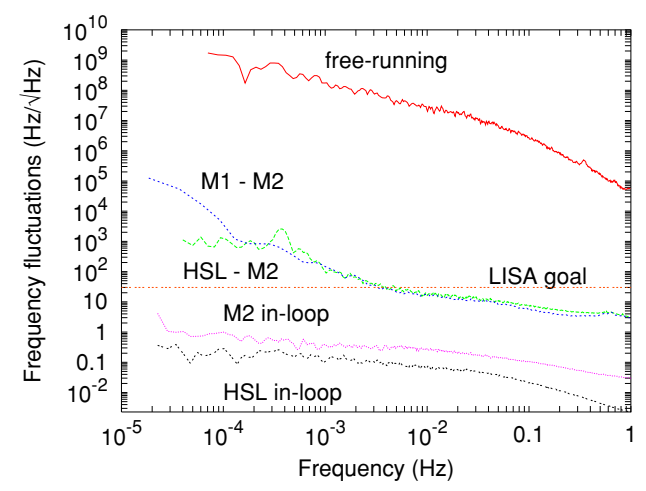

Figure 6. Measured frequency fluctuations.

optical carrier frequency (12 $\mathrm{MHz}$ for the second system) with a modulation index of 0.5 ( 0.3 for the second system). The light reflected from the cavity was monitored by a fast Si-photodiode with a resonant preamplifier. A loop filter with three damped integrators (with a corner frequency of $100 \mathrm{~Hz}$ ) acted on a PZT mounted on the laser crystal to correct for fast frequency fluctuations. A second control loop used the PZT voltage as error signal and acted on the crystal temperature via its temperature controller to compensate for long-term frequency drifts. The unity gain frequencies of the PZT control loops were measured as $22 \mathrm{kHz}$ for the laser demonstrator and $12.5 \mathrm{kHz}$ for the reference laser.

Figure 6 shows the results obtained from frequency stabilization of the LISA laser demonstrator. The top trace shows the free-running frequency noise of the NPRO-based laser demonstrator. Free running means that the pump diodes were driven by a constant current source and the temperature of the laser crystal mount was actively stabilized. The trace was obtained from the beat note between free-running laser demonstrator and frequency-stabilized reference laser M2.

The trace 'M1-M2' shows the beat note between two identical frequency-stabilized reference lasers (the first laser was labelled M1, the second M2) that was obtained in a setup where laser M1 instead of the laser demonstrator was locked to cavity 1 and M2 was locked to cavity 2 and the beat frequency between both lasers was recorded. This is a measure for the sensitivity of the setup. The difference in frequency between laser demonstrator and M2 has been labelled 'HSL-M2'. This trace is the actual measurement trace and it showed 


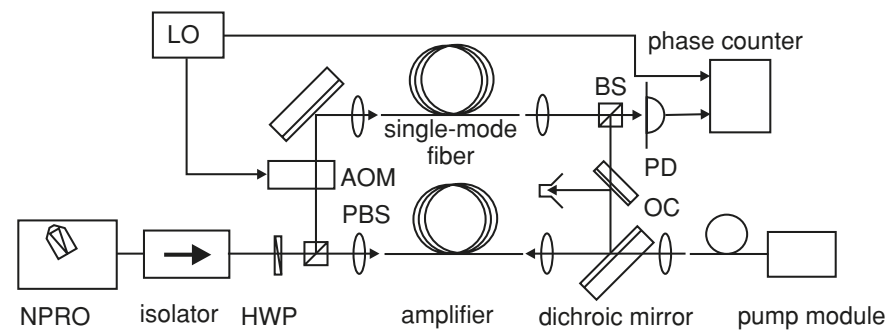

Figure 7. Low-frequency phase noise measurement setup for frequencies up to $0.5 \mathrm{~Hz}$ with phase counter.

essentially the same frequency fluctuations as measured between M1 and M2. The two lowest traces show the frequency fluctuations of $\mathrm{M} 2$ and laser demonstrator relative to their reference cavity, measured at the error points of the frequency stabilizations. Both these in-loop signals were significantly below the frequency fluctuations obtained from the difference frequency measurement, which shows that the frequency stability was not loop-gain limited.

The LISA goal of frequency fluctuations below $30 \mathrm{~Hz} / \mathrm{Hz}^{1 / 2}$ could be demonstrated for frequencies above $3 \mathrm{mHz}$. Between $1 \mathrm{mHz}$ and $3 \mathrm{mHz}$ frequency fluctuations below $200 \mathrm{~Hz} / \mathrm{Hz}^{1 / 2}$ were measured.

\section{Two-stage oscillator, amplifier}

A fibre amplifier seeded by a low-power laser represents a promising laser candidate for LISA. In principle, the frequency (and hence the phase) of the amplifier output in an oscillator amplifier system is determined by the frequency of the oscillator. Frequency fluctuations of the oscillator are followed by the amplifier. Fluctuations of the amplifier pump power, temperature fluctuations or acoustic noise represent other, significantly smaller sources for frequency noise at the amplifier output. We have investigated this additional frequency noise.

The fibre amplifier used $3 \mathrm{~m}$ of Yb-doped single-mode double-clad fibre. It was seeded by an NPRO (Mephisto 800 by Innolight) and it was pumped at a wavelength of $976 \mathrm{~nm}$ counterdirectionally to the seed signal by a Peltier-cooled and fibre-coupled pump diode (DFx03 by Dilas). To monitor the optical seed power, a fibre coupler has been incorporated into the input port of the amplifier. All fibre ends were angle polished by $8^{\circ}$ to avoid optical back reflections. In the experiments $1 \mathrm{~W}$ of output power has been generated from $10 \mathrm{~mW}$ of seed power.

Heterodyne Mach-Zehnder interferometers have been used for the excess frequency noise measurements. The setup in figure 7 has been used for the low-frequency measurements from $10^{-4} \mathrm{~Hz}$ up to $0.5 \mathrm{~Hz}$. Light of a $1064 \mathrm{~nm}$ NPRO (Mephisto 800 by Innolight) was shielded from backreflections by an optical isolator. The subsequent half-wave plate (HWP) in combination with the polarizing beam splitter (PBS) was used to adjust the power distribution in the two interferometer arms. One arm contained the fibre amplifier, the other arm contained an acousto-optic modulator (AOM) driven by a $40 \mathrm{MHz}$ local oscillator (LO). The light of both arms was brought to interference at a beam splitter (BS) and detected by an InGaAs photodiode (PD). In order to reduce the influence of seed laser frequency fluctuations on the interferometer output phase, another angle-polished single-mode fibre was inserted in the other arm after the AOM such that the effective armlength difference was $\approx 2 \mathrm{~m}$ (limited by available fibre cables). A phase counter (53132A by Agilent) was used to measure the phase between 


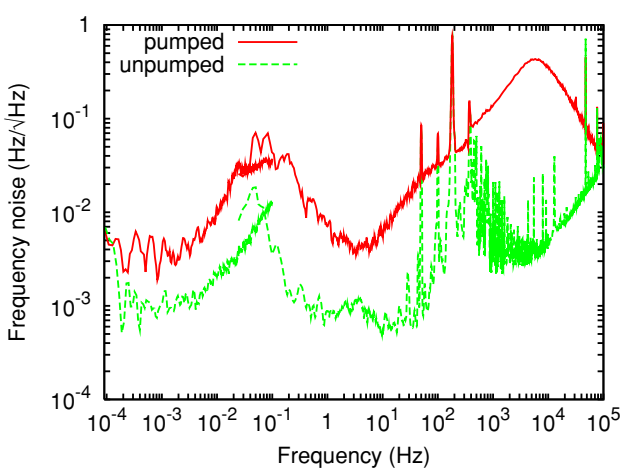

Figure 8. Combined phase noise measurements of the fibre amplifier scaled as frequency noise.

the $40 \mathrm{MHz}$ signal from the LO and the $40 \mathrm{MHz}$ signal from the PD with the advantage that the interferometer does not need to be locked. Locking the interferometer over many hours would require an actuator with a large dynamic range which was not available. Since the phase counter could be read out at $1 \mathrm{~Hz}$ output data rate, spectral estimates up to the Nyquist frequency of $0.5 \mathrm{~Hz}$ could be obtained. The possible effect of aliasing has been estimated to less than $0.14 \mathrm{rad} / \mathrm{Hz}^{1 / 2}$ using the high-frequency measurements described below and found to be negligible.

For the high-frequency measurements the interferometer was locked to constant output phase. The drawback is that the dynamic range of the PZT mirror limited the measurement time to a few minutes. In the setup (not shown), the interferometer output signal was mixed down with the local oscillator signal driving the AOM and fed back to a PZT-driven mirror in one interferometer arm. Either actuator or error signal were recorded. A detailed description is given in [11].

The measured phase noise data have been converted from phase noise $S_{\phi}(f)$ in units of $\mathrm{rad} / \mathrm{Hz}^{1 / 2}$ to frequency noise $S_{v}(f)$ in units of $\mathrm{Hz} / \mathrm{Hz}^{1 / 2}$ by $S_{v}(f)=f \cdot S_{\phi}(f)$. The resulting frequency noise spectral densities have been plotted in figure 8. Despite having been measured with two different setups, the two sets of measurements fit well together. The highest excess frequency noise of the fibre amplifier has been measured as $0.4 \mathrm{~Hz} / \mathrm{Hz}^{1 / 2}$ at $6 \mathrm{kHz}$.

\section{Summary}

The two most promising configurations for the LISA laser are a stand-alone diode-pumped nonplanar ring oscillator (NPRO) or a fibre amplifier seeded by a low-power NPRO.

A laboratory version of the stand-alone laser source was stabilized in frequency to a ULE cavity and in power to an electronic reference. For the first time the LISA requirement of relative power noise below $2 \times 10^{-4} / \mathrm{Hz}^{1 / 2}$ could be fulfilled in the whole frequency range from $0.1 \mathrm{mHz}$ to $1 \mathrm{~Hz}$. In order to characterize the frequency noise properties, beat measurements to an independently stabilized laser were performed. The LISA requirement of frequency noise below $30 \mathrm{~Hz} / \mathrm{Hz}^{1 / 2}$ could be achieved for frequencies above $3 \mathrm{mHz}$.

The excess frequency noise produced by the fibre amplifier in the LISA measurement window was below $0.1 \mathrm{~Hz} / \mathrm{Hz}^{1 / 2}$, which is negligible compared to the free-running frequency fluctuations of a free-running NPRO but above the $\mu \mathrm{Hz} / \mathrm{Hz}^{1 / 2}$ frequency noise requirement of LISA. If an oscillator amplifier system is to be used for LISA, the measured excess noise has to be considered depending on the implementation. 


\section{Acknowledgments}

Part of this work was supported by the European Space Agency in the 'High Stability Laser' project, and part of this work was supported by Deutsche Forschungsgemeinschaft through SFB 407.

\section{References}

[1] Danzmann K and Rüdiger A 2003 LISA technology-concepts, status, prospects Class. Quantum Grav. $20 \mathrm{~S} 1-9$

[2] McNamara P W, Ward H, Hough J and Robertson D 1997 Laser frequency stabilisation for spaceborne gravitational wave detectors Class. Quantum Grav. 14 1543-7

[3] Sheard B S, Gray M B, McClelland D E and Shaddock D A 2003 Laser frequency stabilization by locking to a LISA arm Phys. Lett. A 320 9-21

[4] Tinto M, Estabrook F B and Armstrong J W 2002 Time-delay interferometry for LISA Phys. Rev. D 65 082003-1-12

[5] Kane T J and Byer R L 1985 Monolithic, unidirectional single-mode Nd:YAG ring laser Opt. Lett. 10 65-7

[6] Freitag I, Tünnermann A and Welling H 1995 Power scaling of diode-pumped monolithic Nd:YAG lasers to output powers of several watts Opt. Commun. 115 511-5

[7] Rugi E, Mueller P and Lambelet P 2003 Scalable high brightness laser pump for aerospace applications European Conference on Lasers and Electro Optics (CLEO/Europe)

[8] LISA: System and Technology Study Report ESA document ESA-SCI 112000

[9] Drever R W P, Hall J L, Kowalski F V, Hough J, Ford G M, Munley A J and Ward H 1983 Laser phase and frequency stabilization using an optical resonator Appl. Phys. B 31 97-105

[10] Black E D 2001 An introduction to Pound-Drever-Hall laser frequency stabilization Am. J. Phys. 69 79-87

[11] Tröbs M, Weßels P and Fallnich C 2005 Power- and frequency-noise characteristics of an Yb-doped fiber amplifier and actuators for stabilization Opt. Exp. 13 2224-35 